\title{
Compactly Supported Biorthogonal Wavelet Bases on the Body Centered Cubic Lattice
}

\author{
J. J. Horacsek, U. R. Alim ${ }^{\dagger}$ \\ Department of Computer Science, University of Calgary, Calgary AB, Canada
}

\begin{abstract}
In this work, we present a family of compact, biorthogonal wavelet filter banks that are applicable to the Body Centered Cubic (BCC) lattice. While the BCC lattice has been shown to have superior approximation properties for volumetric data when compared to the Cartesian Cubic (CC) lattice, there has been little work in the way of designing wavelet filter banks that respect the geometry of the BCC lattice. Since wavelets have applications in signal de-noising, compression, and sparse signal reconstruction, these filter banks are an important tool that addresses some of the scalability concerns presented by the BCC lattice. We use these filters in the context of volumetric data compression and reconstruction and qualitatively evaluate our results by rendering images of isosurfaces from compressed data.
\end{abstract}

Categories and Subject Descriptors (according to ACM CCS): I.3.5 [Computer Graphics]: Computational Geometry and Object ModelingSplines G.1.2 [Mathematics of Computing]: Approximation-Wavelets and fractals

\section{Introduction}

Wavelet bases provide a powerful framework for signal analysis and allow one to decompose a signal into a sparse representation through a set of iterated filter banks. In visualization, this transformation affords many applications such as noise reduction, compression via sub-band coding, and sparse reconstruction. In one dimension, the analysis of multichannel filter banks is well understood; this is likely due to the fact that the analysis boils down to the manipulation of Laurent polynomials which form a principal ideal domain, so one may use the properties of unique polynomial division to derive easily parametrizable filter banks.

The multivariate case is considerably more complex. The most simple extension to multiple dimensions is the separable extension. i.e. extending univariate techniques via tensor product along the cardinal axes. This leads to a dyadic scheme that "shaves" off high frequency content along the cardinal axes. While this is a very natural approach, it treats high frequencies unequally; it partitions the fundamental region of the lattice into hyper-cubic subregions. Therefore, regions that are captured about the diagonal of this hypercube are treated in the same way as regions that are captured about the cardinal directions.

Of course there is the more general situation in which the wavelet filter banks are non-separable. In this case, a general sub-sampling matrix is chosen which affects both the distribution of points which are thrown

\footnotetext{
$\dagger$ \{joshua.horacsek,ualim\}@ucalgary.ca
}

out as a result of the sub-sampling process as well as the dilation of the resulting point-set back to the original point distribution. Filters must be then constructed so that certain multivariate reconstruction criteria are satisfied (e.g. perfect reconstruction or the quadrature mirror filter criteria). In multiple dimensions, this is difficult due to the lack of a unique factorization within the multivariate polynomial ring.

When data are sampled according to a non-Cartesian lattice, care should be taken so as to design filter banks that respect the geometry of that lattice. For example, one can use any filter bank designed for $n$-dimensions on any lattice via a linear transformation, however, this may distort the fundamental region of the signal in the frequency domain, resulting in an accompanying distortion of high (and low) frequency content. For hexagonally sampled data in two dimensions, there exist a few works that successfully derive filter banks that respect the rotational symmetry of the hexagonal lattice [CS93, CSW92].

Processing data on a non-Cartesian lattice is not done without good reason. It is well known that a hexagonal lattice in two dimensions can represent a function with the same fidelity as a Cartesian lattice with up to $14 \%$ fewer samples. In three dimensions, the optimal lattice is the body centered cubic (BCC) lattice, which brings a potential $30 \%$ saving as compared to the Cartesian cubic (CC) lattice. Data processing on the BCC lattice has slowly been studied over the past few years, but surprisingly there do not exist wavelet constructions that respect the symmetry of the $\mathrm{BCC}$ lattice. This is an important component in data processing since a sparse representation is key to many data processing applications.

In this work, we consider the dyadic biorthogonal case. Our work can be seen as an extension to the work of Cohen et al. [CS93] who derived compactly supported biorthogonal wavelet bases for the $2 \mathrm{D}$ hexagonal lattice. We slightly modify their construction procedure and extend it to $3 \mathrm{D}$ to derive compactly supported biorthogonal wavelet bases that respect the symmetry of the BCC lattice. In our design methodology, 

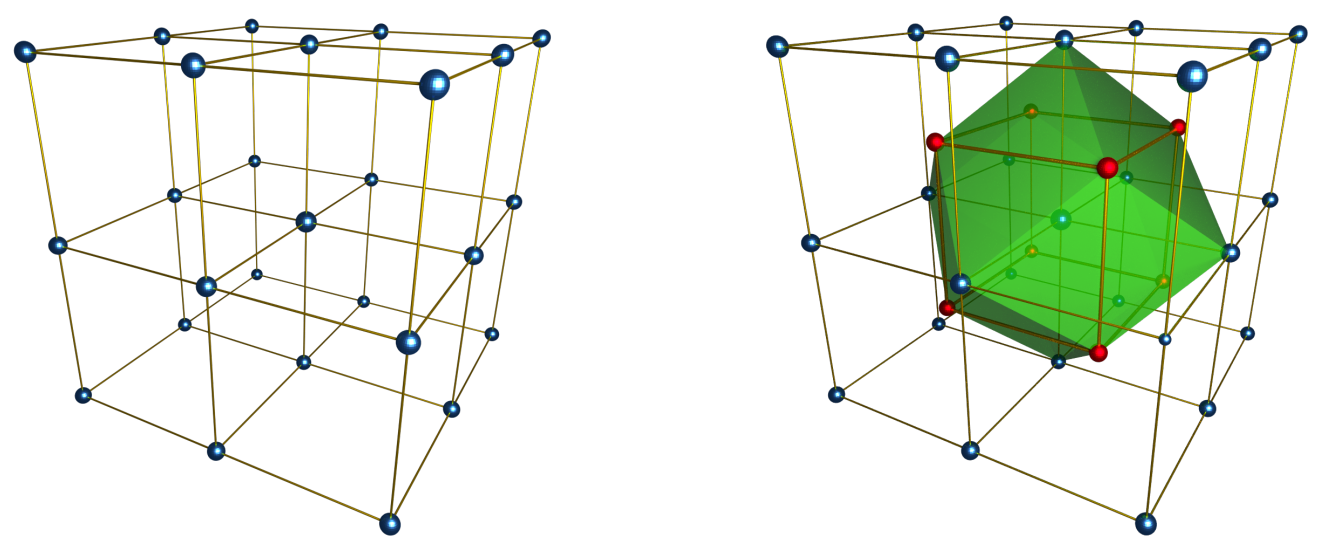

Figure 1: The Cartesian (CC) and Body Centered Cubic (BCC) lattice, left and right respectively. Notice the coset structure of the BCC lattice, denoted by the red and blue node colouring. The support of the linear box spline on the BCC lattice is shown by the green rhombic dodecahedron.

the high-pass filters are first chosen with a specific (and symmetric) geometry; these high-pass filters lead to a dual low pass filter that corresponds to the discrete linear box spline [EVDVM08]. We also use the boot-strapping procedure of Cohen et al. [CS93] to derive higher order wavelets. Finally, we look at the frequency responses of our wavelets in the Fourier domain, and compare them to wavelets on the Cartesian lattice quantitatively and qualitatively in the context of volumetric data compression (via thresholding) and iso-surface rendering.

The main contribution of this work is therefore the design of a practical wavelet scheme for volumetric data that is sampled on the BCC lattice. While other works have peripherally touched upon this topic, an explicit construction scheme on the BCC lattice has so far remained elusive. Even though our construction is based on the work of Cohen et al. [CS93], the extension to BCC is non-trivial. In particular, it is not obvious how to choose starting filters so that the low pass filter yields a box spline. We show that a particular family of high pass filters aligned in the Voronoi relevant directions of the BCC lattice - akin to the $D 3 b Q 15$ discretization of the velocity space in the lattice Boltzmann method [AEM09] — does in fact lead to a low pass filter that corresponds to the linear box spline. Our presentation is self-contained and reviews the necessary mathematical background behind dyadic non-Cartesian multiresolution analysis so as to stimulate further research on this topic. For the benefit of the practitioner, we also provide the filter weights corresponding to the linear and quintic box spline wavelet bases.

\section{Background}

\subsection{Related Work}

Within the context of scientific visualization, the BCC lattice has been subject to much exploration from researchers and has found applications in problems ranging from computed tomography [FAVM09] and fluid simulation [AEM09] to surface reconstruction [HK15] and frameless volume visualization [PK16]. A significant body of literature related to the BCC lattice has focused on the representation and reconstruction of data. Reconstruction is a non-trivial problem because, when working with data on the BCC lattice, it is no longer possible to use the ubiquitous tensor product B-splines to reconstruct data between sample values. However, various reconstruction filters have been designed to work on the
BCC lattice, often with smaller support than their Cartesian counterparts. For example, the linear and quintic box splines of Entezari et al. have generally smaller support than the tensor product linear and cubic B-splines on the Cartesian lattice [EDM04,EVDVM08]. Kim presented a quartic box spline with even smaller support but the same smoothness as the quintic box spline, as well as a tensor product style box spline (with larger support but reasonable evaluation speed due to the structure of the spline) [Kim13]. There are also DC-splines [DC10, Csé13, AO15], which attempt to bring the advantages of tri-linear interpolation to the BCC lattice; we suspect these are not a new family of splines, but rather a sub-family of the exponential box splines [Ron88].

It is often necessary to apply discrete filters to continuous data that are represented in a discrete fashion via lattice samples. Towards this end, the discrete filters should be carefully designed so as to match the approximation capabilities of the continuous reconstruction filter. Alim et al. designed discrete derivative filters for the BCC lattice [AMC10, HAM11], and proposed a fast discrete Fourier transform for BCC data [AM09]. In terms of multiresolution approaches on the BCC lattice, there is not much work. Alim et al. proposed a Cartesian to BCC downsampling strategy that preserves the quality of the fine level at the coarse level [AO15]. There is also the hierarchical scheme of Entezari et al. which downsamples data from an original Cartesian lattice to a face centered cubic (FCC) lattice (two-fold reduction), a BCC lattice (four-fold reduction), and a coarse Cartesian lattice (eight-fold reduction) [EMBM06]. However, the paper really only tells half of the bigger picture; it low-passes data, but there are no corresponding high-pass filters. Operations such as compression and noise reduction are therefore out of reach of this technique. Some work, however, has gone into finding dilation matrices for non-dyadic wavelet transforms on the BCC lattice [EMV04], but these have yet to yield any filter banks that we know of. This is likely because it is difficult to design filter-banks with more than two channels. Kovacevic et al. provide an in-depth and general characterization of the relationship between the dual and primal filters in non-separable filter-bank design [KV92]. However, their methodology is difficult to apply to our case since we desire that symmetry be the constraining factor.

Interestingly, the primal low pass filters derived from our construction leads to a familiar class of box spline wavelets. Box splines naturally appear in the study of multivariate refinable (i.e. wavelet-like) functions, 


\section{J. J. Horacsek \& U.R. Alim / BCC Wavelets}

likely due to their self similarity [DBHR13, MMZ11b]. Box splines have also been studied in connection with dyadic wavelet schemes. He et al. provided a factorization of box spline filters into corresponding high-pass filters and characterized a family of duals for the box spline low pass filter [HL03]. While that work gives an explicit form for a (dyadic) dual of a small class of box splines, the factorization of high-pass filters leads to a design that is not appropriate in practice; the resulting highpass filters are asymmetric and have seemingly random geometry. More troubling is that their parametrization of the dual low pass filter banks appear to lack the same symmetry as the primal low pass filter. Ye et al. proposed a geometric Fourier domain approach to design bivariate wavelet filters [YE12]. While their approach can be extended to BCC, the resulting filters would have infinite support and would need to be applied in the Fourier domain. We prefer the finite support of our design, as this allows us to apply our filters without first transforming the whole input dataset.

Another important aspect of filter design is convergence; if a filter bank does not converge to an appropriate function, it may have practical ramifications on the bound of the coefficients of the resulting decomposition. In the general setting with some dilation matrix $D$, convergence and smoothness of refinable (wavelet-like) functions have been characterized by Jia et al. [Jia99, JJL02]. The more general case of non-linear (data-dependent) refinable functions has also been characterized [MMZ11a, MMZ11b].

\subsection{Data Sampled on the BCC Lattice}

For volumetric visualization of scalar data, we have a function $f: \mathbb{R}^{3} \rightarrow \mathbb{R}$ that we wish to extract information from, perhaps an iso-surface or an image via ray-marching. Often these data are discrete samples located on a Cartesian grid. That is, each sample lies in space at some triple in the set $\mathbb{Z}^{3}$. Data between these points are then interpolated to approximate the underlying function $f(\mathbf{x})$.

While this method is simple, it is suboptimal. A more robust approach allows us to incorporate different sampling patterns, thus an invertible matrix $L$ is introduced, which is often known as the generating matrix for the sampling lattice. A function is now sampled according to the set $L \mathbb{Z}^{3}$. The optimal sampling lattice in $\mathbb{R}^{3}$, the BCC lattice, is generated by the matrix

$$
L=\left[\begin{array}{ccc}
-1 & 1 & 1 \\
1 & -1 & 1 \\
1 & 1 & -1
\end{array}\right]
$$

This is depicted in Figure 1, alongside the CC lattice. The data we concern ourselves with in this work are the samples of some signal $s(\mathbf{x})$ at the lattice sites of the BCC lattice. That is, we work with the sequence

$$
s[\mathbf{n}]:=s(L \mathbf{n}) \text { such that } \mathbf{n} \in \mathbb{Z}^{3} .
$$

It is important to stop and note that this matrix is different than our dilation matrix. In our scheme, $L$ defines the locations of the samples in space, whereas our dyadic dilation matrix is $2 I$.

\subsection{Multiresolution Analysis in $L^{2}(\mathbb{R})$}

At the heart of any wavelet decomposition scheme is the concept of a multiresolution analysis (MRA). A MRA focuses on the representation of a signal at different scales and the associated wavelet filter banks are the tools that allow one to shift between scales. Formally, a dyadic multiresolution analysis over $\mathbb{R}$ consists of the sequence of function spaces $\left\{\cdots V_{-1}, V_{0}, V_{1} \cdots\right\}$. These function spaces are spanned by the dyadic translates and scales of a scaling function $\varphi: \mathbb{R} \rightarrow \mathbb{R}$. That is, $V_{j}$ consists of all functions

$$
V_{j}:=\left\{\sum_{i \in \mathbb{Z}} c_{i j} \varphi\left(2^{-j} x-i\right)\right\}
$$

where $c_{i j}$ is a coefficient associated to each translated and scaled basis function. A MRA satisfies the following properties:

$$
\begin{gathered}
\{0\} \leftarrow \cdots V_{2} \subset V_{1} \subset V_{0} \subset V_{-1} \subset V_{-2} \subset \cdots \rightarrow L^{2}(\mathbb{R}), \\
f(x) \in V_{j} \Longleftrightarrow f(2 x) \in V_{j-1} \Longleftrightarrow f\left(2^{j} x\right) \in V_{0}, \text { and } \\
\{\varphi(x-k)\} \text { is an orthonormal basis for } V_{0} .
\end{gathered}
$$

We adopt the convention of Cohen et al. [CS93] and denote the low-pass and high-pass filters as $m_{0}$ and $m_{1}$ respectively. In the orthogonal two-channel case, the Fourier transforms of the filters are related by $\hat{m}_{1}=e^{-l \omega} \hat{m}_{0}(\omega+\pi)$ (where we use $l$ to denote the complex unit), and must satisfy

$$
\hat{m}_{0}(0)=1 \text { and }\left|\hat{m}_{0}(\omega)\right|^{2}+\left|\hat{m}_{0}(\omega+\pi)\right|^{2}=1 .
$$

The first condition is necessary for the filter to converge to a wavelet scaling function, while the second (known as the conjugate quadrature filter criterion) ensures that the filter bank achieves perfect reconstruction. The scaling function is then defined via the infinite product

$$
\hat{\varphi}(\omega)=\prod_{j=1}^{\infty} \hat{m}_{0}\left(2^{-j} \omega\right)
$$

which comes about as the consequence of the two scale relationship between the function spaces $V_{j}$. If these products converge point-wise to a function in $L^{2}(\mathbb{R})$, then we have a MRA. The wavelet function can also be expressed as

$$
\hat{\psi}(\omega)=\hat{m}_{1}\left(\frac{\omega}{2}\right) \hat{\varphi}\left(\frac{\omega}{2}\right),
$$

or equivalently in the spatial domain as

$$
\psi(x / 2)=\sum_{i} m_{1}[i] \varphi(x-i) .
$$

Biorthogonal wavelets provide a more general framework that allows a higher degree of freedom when designing filter banks. In this paradigm, we now have a pair of dual low-pass filters $m_{0}$ and $\tilde{m}_{0}$ which must satisfy

$$
\begin{gathered}
\hat{m}_{0}(0)=\hat{\tilde{m}}_{0}(0)=1, \hat{m}_{0}(\pi)=\hat{\tilde{m}}_{0}(\pi)=0 \text { and } \\
\overline{\hat{m}_{0}(\omega)} \hat{\tilde{m}}_{0}(\omega)+\overline{\hat{m}_{0}(\omega+\pi)} \hat{\tilde{m}}_{0}(\omega+\pi)=1 .
\end{gathered}
$$

The latter equation is the Fourier domain representation of the dyadic biorthogonality constraint

$$
\left(\bar{m}_{0} * \tilde{m}_{0}\right)[2 i]=\delta[i],
$$

where $\bar{m}_{0}[i]:=m_{0}[-i]$ and $\delta[\cdot]$ denotes the Kronecker delta sequence. The high-pass filters that achieve perfect reconstruction are given by

$$
\hat{m}_{1}(\omega)=e^{-l \omega} \overline{\overline{\tilde{m}}_{0}(\omega+\pi)} \text { and } \hat{\tilde{m}}_{1}(\omega)=e^{-l \omega} \overline{\hat{m}_{0}(\omega+\pi)} .
$$

The pair of low-pass filters have the associated scaling functions

$$
\hat{\varphi}(\omega)=\prod_{j=1}^{\infty} \hat{m}_{0}\left(2^{-j} \omega\right) \text { and } \hat{\tilde{\varphi}}(\omega)=\prod_{j=1}^{\infty} \hat{\tilde{m}}_{0}\left(2^{-j} \omega\right)
$$

and the wavelet functions are

$$
\hat{\psi}(\omega)=m_{1}\left(\frac{\omega}{2}\right) \hat{\varphi}\left(\frac{\omega}{2}\right) \text { and } \hat{\tilde{\psi}}(\omega)=\hat{\tilde{m}}_{1}\left(\frac{\omega}{2}\right) \hat{\tilde{\varphi}}\left(\frac{\omega}{2}\right) .
$$




\subsection{Box Splines}

Box splines are multivariate piecewise polynomial functions that are analogous to the univariate B-splines [DBHR13]. They can be obtained by successively convolving a box function along different direction vectors. In particular, we are interested in the linear box spline [EDM04] that is analogous to the Courant element in two dimensions; it very naturally reflects the geometry of the BCC lattice (Figure 1). While the linear box spline has a simple piecewise polynomial representation, the Fourier domain representation is even more simple,

$$
c(\boldsymbol{\omega}):=\left(\frac{1-e^{\imath \omega_{0}}}{l \omega_{0}}\right)\left(\frac{1-e^{\imath \omega_{1}}}{l \omega_{1}}\right)\left(\frac{1-e^{\imath \omega_{2}}}{l \omega_{2}}\right)\left(\frac{1-e^{-\imath\left(\omega_{0}+\omega_{1}+\omega_{2}\right)}}{-l\left(\omega_{0}+\omega_{1}+\omega_{2}\right)}\right) .
$$

It is also well known that the linear box spline can be obtained from iterating the discrete filter

$$
C(\mathbf{z}):=\left(\frac{1+z_{0}}{2}\right)\left(\frac{1+z_{1}}{2}\right)\left(\frac{1+z_{2}}{2}\right)\left(\frac{1+z_{0}^{-1} z_{1}^{-1} z_{2}^{-1}}{2}\right)
$$

about the two-scale relationship [DBHR13]. The linear box spline can be convolved with itself to yield the higher-order quintic box spline [EDM04].

\section{Wavelets on the BCC Lattice}

\subsection{Dyadic Biorthogonal Filterbanks on the BCC Lattice}

Moving away from the single variable case, we consider filters located at BCC lattice sites. A dyadic scheme in three variables corresponds to an 8-channel filter bank; in general this is related to the coset vectors of the dilation matrix, which is also related to the determinant of the dilation matrix [KV92]. For a dyadic scheme in three dimensions, we have the dialation matrix $2 I$ with $\operatorname{det} 2 I=8$. Thus, we introduce the 8 filters $m_{0}, \ldots, m_{7}$ and their duals $\tilde{m}_{0}, \ldots, \tilde{m}_{7}$. Our goal is to construct a family of these filter banks that respect the geometry of the BCC lattice and achieve perfect reconstruction.

We cement the notation that the discrete filter $m[\mathbf{n}]$ has the discrete Fourier transform and Z-transform

$$
\hat{m}(\boldsymbol{\omega})=\sum_{\mathbf{n} \in \mathbb{Z}^{3}} m[\mathbf{n}] e^{-\imath \boldsymbol{\omega}^{T} L \mathbf{n}} \text { and } M(\mathbf{z})=\sum_{\mathbf{n} \in \mathbb{Z}^{3}} m[\mathbf{n}] \mathbf{z}^{-\mathbf{n}}
$$

respectively. Note that the Fourier form can be obtained from the Z-transform via

$$
\hat{m}(\boldsymbol{\omega})=M\left(e^{\imath \boldsymbol{\omega}^{T} L}\right) .
$$

Recall that $\hat{m}(\boldsymbol{\omega})$ is a periodic function with respect to the dual face-centered cubic (FCC) lattice that is generated by the matrix $L^{-T}$.

We use the notation $\stackrel{\circ}{m}[\mathbf{n}]$ to denote the spatially flipped version of $m$, i.e. $\stackrel{\circ}{m}[\mathbf{n}]=m[-\mathbf{n}]$. We also use the notation $\bar{a}$ to denote the complex conjugate of $a$. Note that $\widehat{\hat{m}}(\boldsymbol{\omega})=\overline{\hat{m}(\boldsymbol{\omega})}$. We also define the aliasing vectors $\boldsymbol{\pi}_{0}:=$ $(0,0,0)^{T}, \boldsymbol{\pi}_{1}:=(0,0, \pi)^{T}, \boldsymbol{\pi}_{2}:=(0, \pi, 0)^{T}, \boldsymbol{\pi}_{3}:=(0, \pi, \pi)^{T}, \boldsymbol{\pi}_{4}:=(\pi, 0,0)^{T}$, $\boldsymbol{\pi}_{5}:=(\pi, 0, \pi)^{T}, \boldsymbol{\pi}_{6}:=(\pi, \pi, 0)^{T}$ and $\boldsymbol{\pi}_{7}:=(\pi, \pi, \pi)^{T}$. Observe that for a dyadically downsampled sequence $x[\mathbf{n}]:=m[2 \mathbf{n}]$, we have

$$
\hat{x}(\boldsymbol{\omega})=\frac{1}{8} \sum_{i=0}^{7} \hat{m}\left(\frac{\boldsymbol{\omega}}{2}+L^{-T} \boldsymbol{\pi}_{i}\right) \text { and } X(\mathbf{z})=\frac{1}{8} \sum_{i=0}^{7} M\left(\sqrt{\mathbf{z}} e^{\imath \boldsymbol{\pi}_{i}}\right),
$$

and for an up-sampled sequence $y[\mathbf{n}]:=\{m[\mathbf{n} / 2]$ if $\mathbf{n}$ is all even, and 0 otherwise $\}$, we have

$$
\hat{y}(\boldsymbol{\omega})=\hat{m}(2 \boldsymbol{\omega}) \text { and } Y(\mathbf{z})=M\left(\mathbf{z}^{2}\right) .
$$

\subsection{Perfect Reconstruction}

At a high level, the perfect reconstruction sub-band encoding scheme guarantees that, after a signal has been broken down into its low and high pass frequencies it can be re-assembled without introducing any aliasing. This is shown diagrammatically in Figure 2; the equivalent Fourier and Z-transform representations are

$$
\hat{s}^{\prime}(\boldsymbol{\omega})=\sum_{j} \sum_{i} \hat{s}\left(\boldsymbol{\omega}+L^{-T} \boldsymbol{\pi}_{i}\right) \overline{\hat{m}_{j}\left(\boldsymbol{\omega}+L^{-T} \boldsymbol{\pi}_{i}\right)} \hat{\tilde{m}}_{j}(\boldsymbol{\omega}),
$$

and

$$
S^{\prime}(\mathbf{z})=\sum_{j} \sum_{i} S\left(\mathbf{z} e^{\imath \pi_{i}}\right) M_{j}\left(\mathbf{z}^{-1} e^{-\imath \boldsymbol{\pi}_{i}}\right) \tilde{M}_{j}(\mathbf{z}) .
$$

respectively. Since it is easier to work with polynomial matrix equations in computer algebra systems, we mainly adhere to the Z-domain interpretation from this point on. Using Equation (23), it is easily seen that the sub-band coding scheme pictured in Figure 2 will achieve perfect reconstruction if it satisfies the following equations:

$$
\sum_{j} M_{j}\left(\mathbf{z}^{-1} e^{-\imath \boldsymbol{\pi}_{i}}\right) \tilde{M}_{j}(\mathbf{z})=\delta[i], \text { for } i \in\{0, \ldots, 7\} .
$$

To compact notation, we define the following matrix and column vector. Let $\boldsymbol{A}(\mathbf{z})$ be the matrix with the entries defined by $\mathbf{a}_{i, j}(\mathbf{z}):=M_{j}\left(\mathbf{z}^{-1} e^{-i \pi_{i}}\right)$, and let $\boldsymbol{b}(\mathbf{z})$ be the column vector with entries $b_{j}:=\tilde{M}_{j}(\mathbf{z})$. With this notation, we can write the perfect reconstruction criteron (Equation 24) as

$$
\boldsymbol{A}(\mathbf{z}) \boldsymbol{b}(\mathbf{z})=(1,0,0,0,0,0,0,0)^{T} .
$$

Since $\boldsymbol{A}(\mathbf{z})$ is a finite matrix with finite Laurent polynomials, if we know a suitable set of primal filters, we can find the dual filters by inverting $\boldsymbol{A}(\mathbf{z})$ and finding $\boldsymbol{b}(\mathbf{z})$. However, we still lack a starting point for $\boldsymbol{A}(\mathbf{z})$. The next proposition gives a way of peeking into a solution given a "reasonable" set of starting dual high pass filters.

Proposition 3.1 If the set of filters $m_{0}, \ldots, m_{7}, \tilde{m}_{0}, \ldots, \tilde{m}_{7}$ has perfect reconstruction, then

$$
\tilde{M}_{0}(\mathbf{z})=k \mathbf{z}^{\mathbf{n}} \boldsymbol{A}_{0,0}(\mathbf{z})
$$

where $\boldsymbol{A}_{0,0}(\mathbf{z})$ is the matrix minor associated with $\tilde{M}_{0}(\mathbf{z}), k \neq 0, \mathbf{n} \in \mathbb{Z}^{3}$, and

$$
\sum_{i=0}^{7} M_{0}\left(\mathbf{z}^{-1} e^{-i \pi_{i}}\right) \tilde{M}_{0}\left(\mathbf{z} e^{i \pi_{j}}\right)=1
$$

Proof Equation 26 can be derived by using Cramer's rule to solve for the first component of $\boldsymbol{b}(\mathbf{z})$, i.e. the dual low-pass filter $\tilde{M}_{0}(\mathbf{z})$, and using the fact that $\operatorname{det}(\boldsymbol{A}(\mathbf{z}))$ is at most a monomial. Equation 27 comes from expanding $\operatorname{det}(\boldsymbol{A}(\mathbf{z}))$ using cofactor expansion along the rows of $\boldsymbol{A}(\mathbf{z})$ and substituting Equation 26 into the resulting expression.

We remark that Equation 27 is the higher dimensional analog of the dyadic biorthogonality constraint expressed in Equation (11). Similar relationships can be derived for the high-pass filters and their duals.

Thus, if we have a set of primal high pass filters, we can determine the 


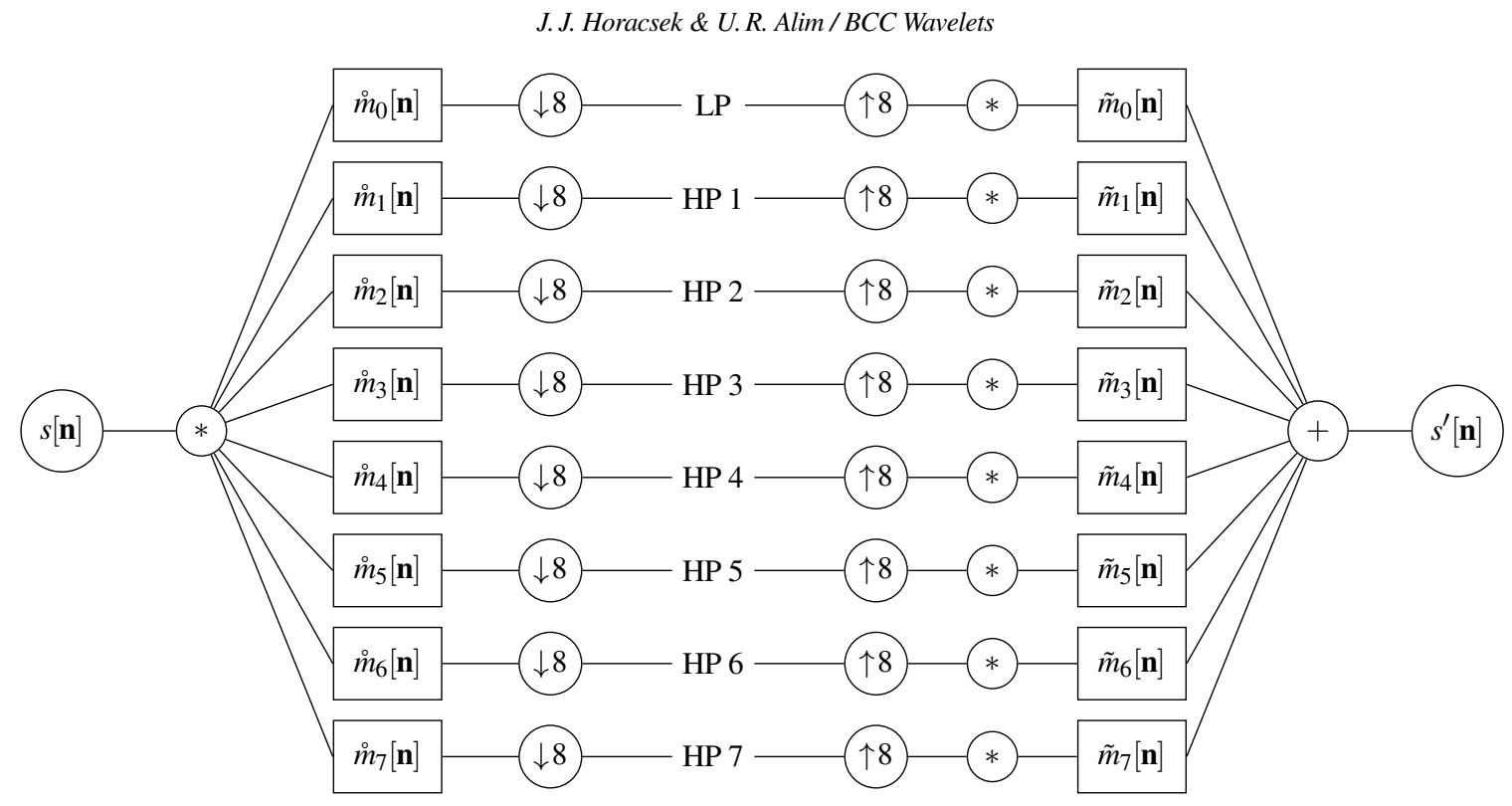

Figure 2: An 8-channel sub-band coding scheme. Here $*$ denotes convolution, $\downarrow 8$ and $\uparrow 8$ denote dyadic down and up sampling respectively. At the end of the reconstruction, the signal must be scaled by 8 .

dual low pass filter, and as a result, the primal low pass filter. Our strategy will be to choose a set of reasonable high pass filters that yield a low pass filter that corresponds to the linear box spline scaling functions. We then derive a dual low pass filter, and complete the system of equations 25 .

\section{Construction}

As previously stated, our wavelet scheme has more than two channels, and the relationship between high and low pass filters is no longer as simple as it is in the dyadic univariate case. While it is not necessary to know the high pass filters a priori, attempting to design the high pass filters from just the constraint that the associated low pass be the linear box spline leads to high pass filters that are unacceptable for volumetric data processing [HL03]. We choose to follow a construction similar to the work of Cohen et al. [CS93]. The salient steps of our construction scheme are as follows.

1. Choose the primal high pass filters with appropriate geometry for the BCC lattice.

2. Use Equation (26) to derive the dual low pass filter, with our choice of high pass filters this generates a shifted version of $C(\mathbf{z})$.

3. To enforce the zero-phase condition, shift the primal low pass filter to the origin, and normalize the filter so that $\hat{\tilde{m}}_{0}(\mathbf{0})=1$.

4. Find the primal low pass filter $m_{0}$, and swap the family of filters so that the dual filters are now the primal filters, and the primal filters are now the dual filters.

5. Solve the system of equations (25) to derive the now dual high pass filters.

A few notes about each step are in order. The choice of filters for step 1. need not be chosen with any particular geometry (see [HL03] as an example), however, we choose them to be a particular filter oriented along principal lattice directions, and the cardinal directions. This has shown to be a rather practical starting point in two dimensions on the hexagonal lattice [CS93], and should roughly partition the frequency domain more isotropically as compared to the tensor product Cartesian filter banks.

\begin{tabular}{c||c} 
Filter & Polynomial \\
\hline$m_{1}[\mathbf{n}]$ & $-\frac{1}{2 z_{1} z_{2}}+\frac{1}{4 z_{1}^{2} z_{2}^{2}}+\frac{1}{4}$ \\
$m_{2}[\mathbf{n}]$ & $-\frac{1}{2 z_{0} z_{2}}+\frac{1}{4 z_{0}^{2} z_{2}^{2}}+\frac{1}{4}$ \\
$m_{3}[\mathbf{n}]$ & $-\frac{1}{2 z_{0} z_{1}}+\frac{1}{4 z_{0}^{2} z_{1}^{2}}+\frac{1}{4}$ \\
$m_{4}[\mathbf{n}]$ & $-\frac{1}{4 z_{0}}+\frac{1}{8 z_{0}^{2}}+\frac{1}{8}$ \\
$m_{5}[\mathbf{n}]$ & $-\frac{1}{4 z_{1}}+\frac{1}{8 z_{1}^{2}}+\frac{1}{8}$ \\
$m_{6}[\mathbf{n}]$ & $-\frac{1}{4 z_{2}}+\frac{1}{8 z_{2}^{2}}+\frac{1}{8}$ \\
$m_{7}[\mathbf{n}]$ & $\frac{1}{8} z_{0}^{2} z_{1}^{2} z_{2}^{2}-\frac{1}{4} z_{0} z_{1} z_{2}+\frac{1}{8}$
\end{tabular}

Table 1: The Z-domain polynomials of all filters given in Figure 3. The first three filters correspond to the filters along the cardinal axes, and the last four correspond to the filters about the diagonal axes.

The shift in step 3. is also not completely necessary, but the zero-phase condition is useful for (volumetric) image processing, as it ensures that fundamental region of the signal is preserved by the low pass filter, and reduces distortion. The duals in step 4. always exist [CS93], and can possibly be derived explicitly, although we choose an algorithmic procedure to derive the dual low pass filters. The swap in step (4.) is valid due to the commutativity of convolution.

\subsection{Choice of High Pass Filters}

Here we present one choice of high pass filters that generates the linear box spline as a low pass filter. This is simply stated in the following proposition.

Proposition 4.1 The filters family of filters $m_{1} \cdots m_{7}$ listed in Table 1 (and partially depicted in Figure 3) generate the linear box spline. 


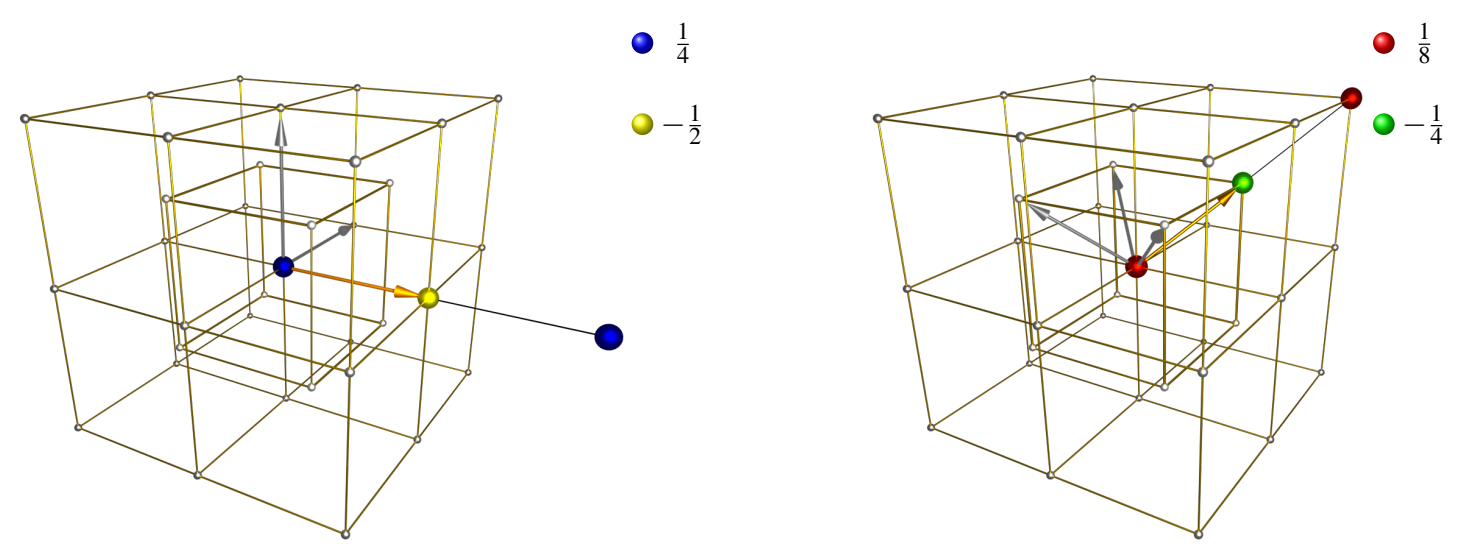

Figure 3: Filter weights for the starting high pass filters. There are two families of filters we start with. $m_{1}$ is depicted in the left sub-figure; it is a simple $1 D$ filter extended along the golden vector. The other two vectors in that image denote the directions along which we extend the same filter to obtain $m_{2}$ and $m_{3}$. Similarly, the rest of the starting high pass filters are given on the right; they are extended along 4 diagonal directions. This gives a total of 7 high-pass channels. Notice how this reflects the symmetries of the BCC lattice; we further remark that the configuration thus obtained is similar to the D3bQ15 discretization of the lattice-Boltzmann method [AEM09].

Proof Complete the system of equations given by 26 , then shift the solution to the origin.

There are other possible choices for these filters. In fact, it is possible to scale them so that they are all the exact same filter extended along each chosen direction, but doing so introduces a seventh square root in each filter coefficient. With the proposed filter weighting scheme, every coefficient's denominator is a power of two, which is computationally more elegant than a seventh root. We could also use different order filters as a starting point for the high pass filters. The chosen high pass filters are a particularly nice starting point though, since it is possible to boot-strap higher regularity wavelets without introducing additional conditions on the starting high pass filters.

\subsection{Computing the Dual Low Pass Filters}

Once the high pass filters are known to converge to the linear box spline, we require a dual low pass filter to complete the dual family of filters. For a given low pass filter, there are infinitely many possible dual filters, a family of such has been characterized explicitly by $\mathrm{He}$ et al. [HL03], however we take a different approach.

We solve for duals algorithmically, similar to the work of Ji et al. [JRS99]. We define a mask $\tilde{M}_{0}$ including the $k^{\text {th }}$ and lower rings of points on the BCC lattice and imbue this with octahedral symmetry. We then substitute this mask into the duality equation and solve the linear system of equations that comes about from matching coefficients. This is implemented with exact arithmetic within Sage [The16], and our coefficients are therefore exact.

\subsection{Boot-strapping Higher Regularity Filters}

The linear case, for certain applications, may provide insufficient regularity; some applications demand at least second order smoothness. We may thus use the boot-strapping procedure of Cohen et al. to design higher regularity wavelets [CS93]. As a note, it may be possible to derive a condition on the initial dual high pass dual filters that guarantee a certain order of regularity of the resulting wavelet bases, but the proposed boot-strap procedure provides a simple scheme that needs no additional theoretical framework to achieve the desired construction, we review it now.

Let $\tilde{m}_{1}^{\prime}, \ldots, \tilde{m}_{7}^{\prime}$ be the family that generates $M_{0}=C(\mathbf{z})$ as a primal low pass filter, and let $p$ and $q$ be positive integers.

1. Find the smallest degree dual to $C^{q+1}(\mathbf{z})$ using the procedure above, call this dual $D(\mathbf{z})$; we thus have $m^{\prime}=c^{q} d$ is dual to $\tilde{m}^{\prime}$

2. Find the primal high pass filters by solving the system of equations (25)

3. Define $m_{j}=\tilde{m}_{j}^{\prime}$, and note that $\tilde{m}_{0}=m_{0}^{\prime}$. Find the dual $D(\mathbf{z})$ to $C(\mathbf{z})^{p+q}$, then set $m_{0}=d c^{p}$ and note that it is dual to $\tilde{m}_{0}$

4. Solve (25) to obtain the unknown filters $\tilde{m}_{j}$

\subsection{Convergence of Scaling Function Bases}

When the dual high-pass filters generate a box spline as the primal low pass filter, then it is well known that the primal scaling function will converge to the continuous box spline. However, depending on the choice of dual, convergence of the dual scaling function is not assured. It is reasonably easy to use the work of Jia et al. to show that our dual filter converges to an $L^{2}$ integrable function for certain $p$ and $q$, although this is not true for all $p$ and $q$ [JJL02]. Convergence is important in practice as it establishes a bound on the coefficients of the wavelet decomposition.

\section{Experiments}

To evaluate the applicability of our filter banks, we perform wavelet thresholding on multiple datasets; namely the Carp, Stent, and Head datasets (shown in Figure 4). We also conducted tests on the Marschner-Lobb (ML) test function [ML94]. We sub-sampled these datasets on a $101 \times 101 \times$ 202 BCC grid, using a cubic tensor product I-MOM to reconstruct the new sample values [BTU01]. We then preformed a seven level hierarchical wavelet decomposition - at each level, the low pass channel of the high level is decomposed into its seven details and one coarse level. We examined the non-zero detail coefficients, sorted them, and removed those below a given threshold so that a certain user-defined percent of the non-zero 


\section{J. J. Horacsek \& U.R. Alim / BCC Wavelets}

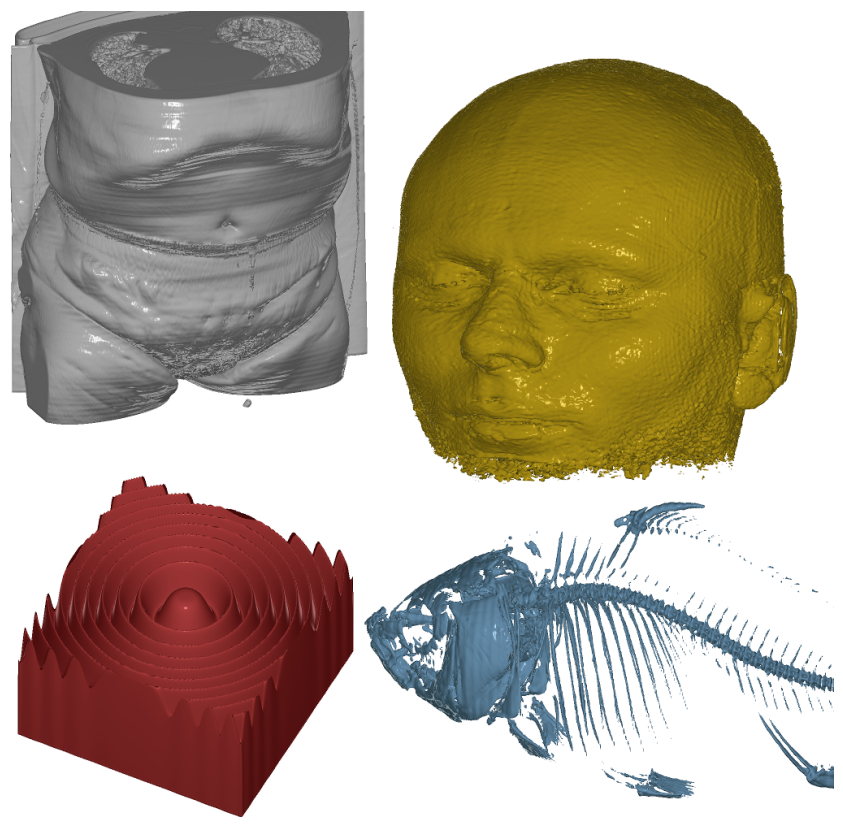

Figure 4: A medley of the original high-resolution datasets. The Stent data set is depicted in gray (original resolution: $512 \times 512 \times 174$, iso-value: 206), Head in yellow (original resolution: $256 \times 256 \times 256$, iso-value: 127.5) Carp in blue (original resolution: $256 \times 256 \times 512$, iso-value: 1435.5), and finally the Marschner-Lobb test function in red (iso-value: 0.5 )

detail coefficients become zero. We then reconstructed the signal and measured the PSNR, defined as $10 \log \left(\max _{I}^{2} / m s e\right)$ where $m s e$ is the integral $\int(f(\boldsymbol{x})-g(\boldsymbol{x}))^{2} d \boldsymbol{x}$ between the approximate reconstruction with fewer detail coefficients $g(\boldsymbol{x})$ and the original high resolution reconstruction $f(\boldsymbol{x})$, and $\max _{I}$ is the maximum possible intensity value for a dataset. We accomplished this via Monte Carlo integration over the volume with $10^{8}$ samples.

As a comparison with wavelets on the Cartesian lattice, we also subsampled these datasets in the same way onto a $128 \times 128 \times 128$ Cartesian lattice, then applied a linear spline wavelet to remove coefficients below a given threshold. The CDF family [CDF92] of wavelets was used as its filters coincide with the starting scaling filters used in our construction procedure. In particular we choose $N=2$ and $\tilde{N}=2$.

To assess how increasing the order of wavelet scheme affects a reconstruction on the $\mathrm{BCC}$ lattice, we performed the bootstrapping procedure with $p=2$ and $q=1$. In the limit, this gives the quintic box spline as a scaling function on the BCC lattice. We then collected the $L^{2}$ error, and compared it with the linear case. We also qualitatively assess the differences in our reconstructions by rendering iso-surfaces of our reconstructed datasets. All reconstructions on the low resolution $\mathrm{CC}$ lattice use the tensor product linear spline, whereas reconstructions on the $\mathrm{BCC}$ use the linear and quintic box splines.

\section{Results}

\subsection{Frequency Responses}

It is worth investigating how our filter banks behave in the Fourier domain. Figure 5 show the frequency response of the linear box spline wavelet filter banks. Note that, on the dual side, the high pass filters are quite simple - this is paralleled by the low pass primal (the box spline) which is also quite simple. Respectively, they have a support size of 3 (each high pass has 3) and 15 lattice sites. The dual low pass has a support of 65 lattice sites, and the primal high pass filters have a support of 77 lattice sites. For $p=2, q=1$ the dual low pass filter has 671 , whereas the primal low pass has 65. The dual high pass has 77, and the primal has 1493 .

\subsection{Comparison}

Figure 6 shows the quantitative results as we add more coefficients. We start with $95 \%$ of the coefficients and add coefficients in increments of $5 \%$. When less data are used, the CC lattice seems to outperform the BCC lattice, however, as we increase the amount of data that are kept, the BCC lattice overtakes the $\mathrm{CC}$ lattice. In a sense this is somewhat analogous to sampling rate of the function, in the under-sampled region, the CC lattice outperforms the BCC lattice. The situation is reversed in the oversampled region. This is consistent with other comparisons between the $\mathrm{BCC}$ and CC lattices since the argument for optimality is in the limit [VCRG14].

We now discuss the qualitative aspects of the reconstructions in Figure 8 and Figure 9. The ML function is interesting since it is oversampled and most of its frequency content is captured very well in the low pass region of the original sampling. It then makes sense that the reconstruction fidelity changes very little as we remove small non-zero detail coefficients. But as we pass the critical frequency range into under-sampling, the $\mathrm{CC}$ lattice overtakes the $\mathrm{BCC}$ lattice. This is fairly consistent in the other datasets as well.

For the Head and Stent datasets, there is severe artifacting when using only $5 \%$ of the data. Between $15 \%$ and $25 \%$ there is minor artifacting around the lips and nose on the Head dataset. On the Stent dataset, we can easily see the effect of crossing the critical frequency point. At 5\% the $\mathrm{CC}$ reconstruction better resembles the original $100 \% \mathrm{CC}$ reconstruction more than the $5 \% \mathrm{BCC}$ resembles the $100 \% \mathrm{BCC}$ reconstruction. However, as we cross that range, the $\mathrm{BCC}$ lattice outperforms the $\mathrm{CC}$ lattice. Finally, the Carp dataset surprisingly does not lose too much fidelity as we remove more data - with only $5 \%$ details the reconstruction is quite faithful to the $100 \%$. One would expect the fine details of the ribs to be lost as the threshold increases.

Moreover, we found that if we increased the order of the family of box spline wavelet we could garner a slight improvement over the linear box spline. This is apparent in the ML PNSR plot, but is not present in the other plots. This is likely due to the fact that the subsampling process band-limits the underlying representation, it is therefore difficult to take advantage of a higher order filter, as there is no additional higher frequencies to capture. Qualitatively, to show the different artifacting caused by the filters, we discarded $95 \%$ of the data and looked at the reconstructions (Figure 7). The artifacts are notably more smooth when using the BCC 2,1 filter. We must say though, increasing the order of the filter bank increases filter size, and there is no guarantee that the increased support will necessarily lead to a more sparse representation, and better compression. 


\section{J. J. Horacsek \& U. R. Alim / BCC Wavelets}
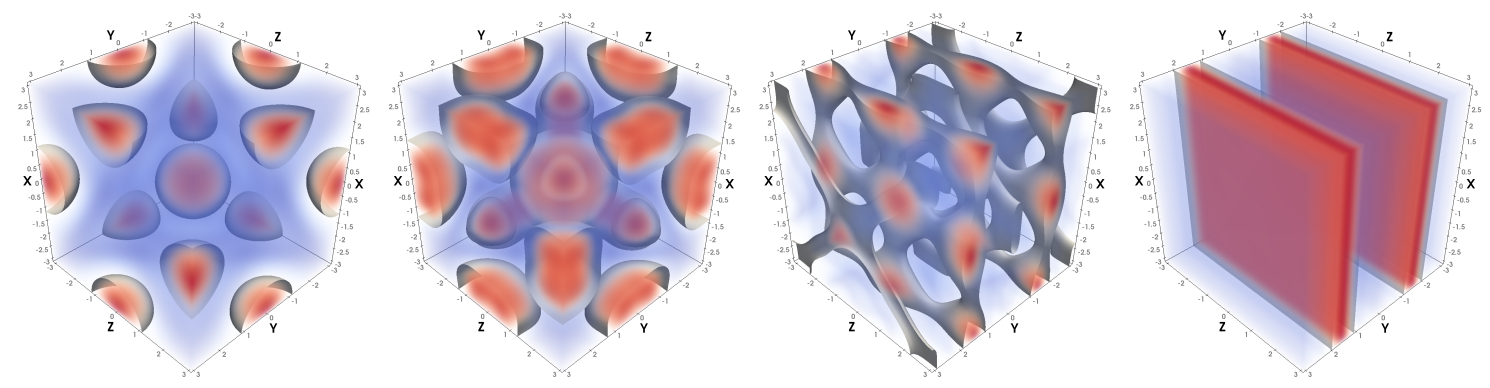

Figure 5: From left to right we have the frequency responses of low pass primal, low pass dual, high pass primal and high pass dual respectively. Note that these are periodized about an FCC lattice, so the fundamental region is replicated in a rhombic dodecahedral pattern. The red indicates the frequencies that will be passed by the filter, whereas blue indicates the frequencies that will be muted. Only one high pass filter is shown here, the rest are symmetric.
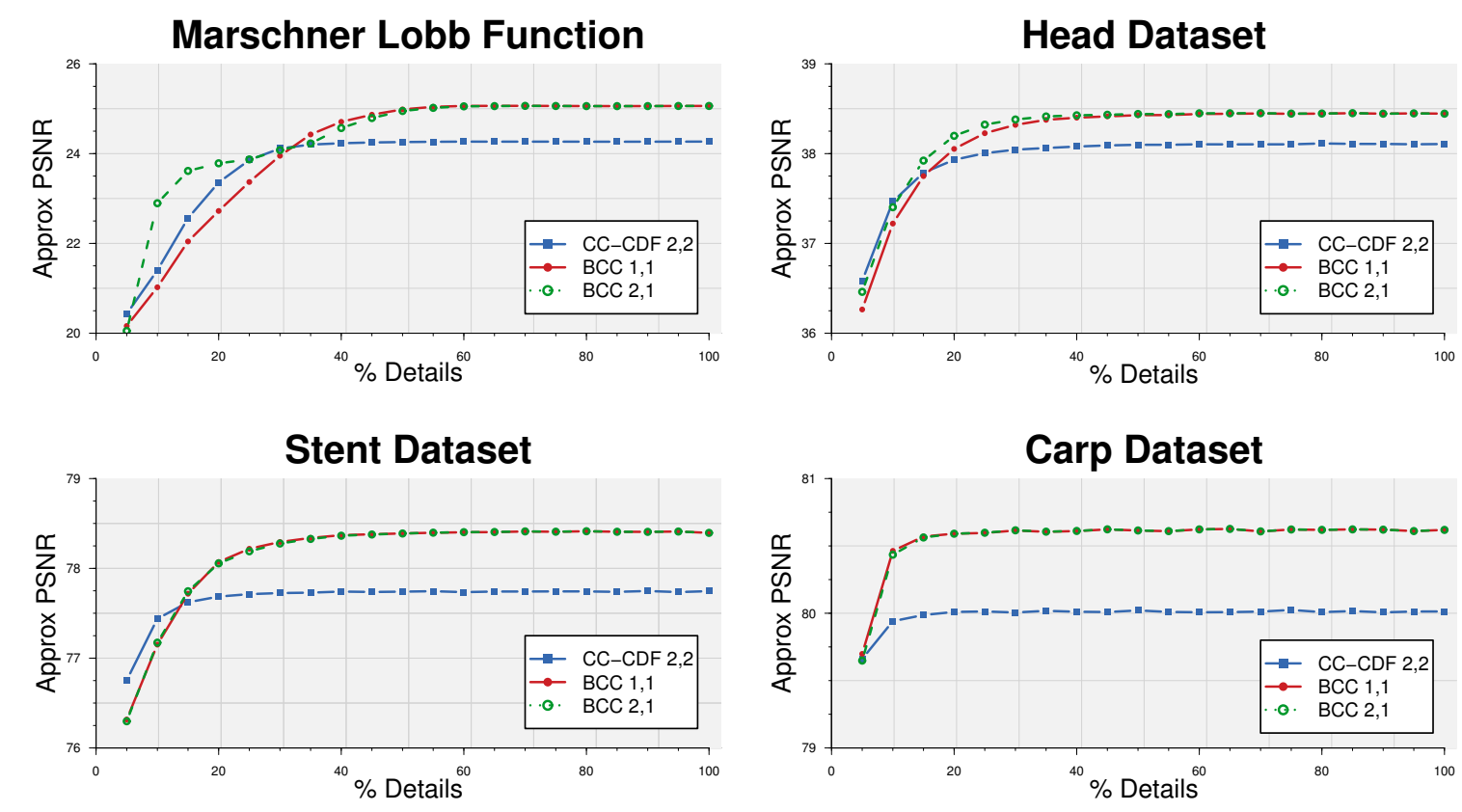

Figure 6: Approximate PNSR for various reconstructions. As the percentage grows, more data are kept, thus the approximation becomes better. Here, higher values on the y-axis indicate a better approximation.

\section{Conclusion}

Since we feel that this is an important tool for those wishing to process data on the BCC lattice, the code to derive these filter banks can be found on GitHub [Hor17]. However, it can be quite computationally expensive to derive these filter banks, so we also provide the filter values, and the boot strapped filter values for $p=2, q=1$ and $p=1, q=2$.

In the future, we would like to explore more elaborate compression schemes on the BCC lattice, in particular exploring blocking schemes, and performing sparse sub-band encoding on those decomposed blocks. Besides the basic interest in compression, it is an interesting question as to what space filling curve is appropriate to use in the necessary run length encoding portion of such schemes. We are also interested in actual wavelet scaling functions as they are the basis for the Wavelet surface reconstruction scheme [MPS08].
We presented a method of constructing a new family of non-separable wavelet filter banks. In particular these filter-banks respect the geometry of the BCC lattice, and evenly distribute the high pass detail coefficients among the high pass channels. We then evaluated these filter banks within the context of compression via thresholding and visualized the results. We were able to throw away much of the data while maintaining visual fidelity and reasonable error.

\section{Acknowledgements}

The authors would like to thank Natural Sciences and Engineering Research Council of Canada and the Department of Computer Science at the University of Calgary for the funding that backed this work. 

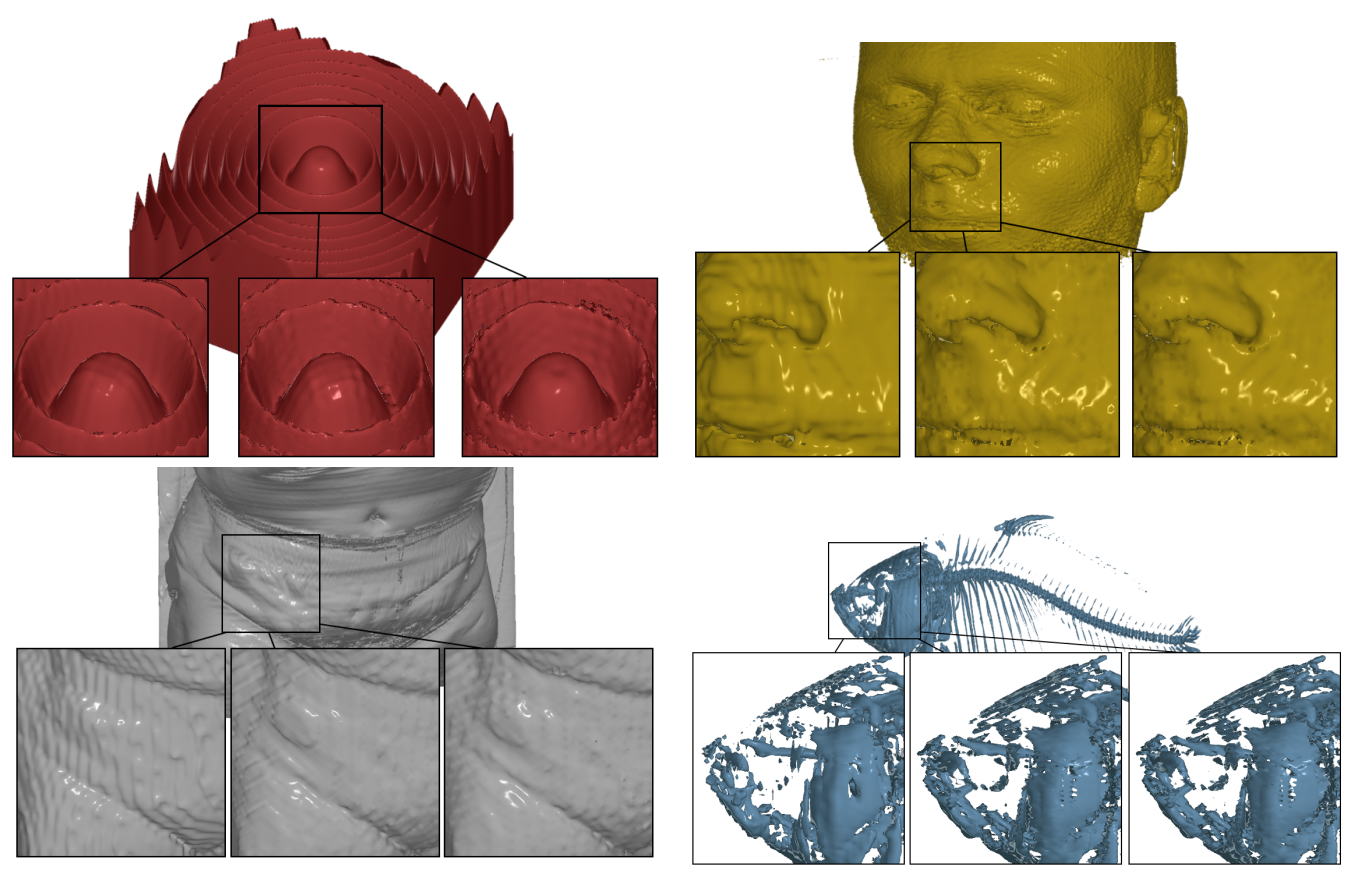

Figure 7: Comparisons with other wavelet thresholding schemes. For the region indicated in each dataset, we show a reconstruction with $95 \%$ of the detail coefficients removed. On the left is the tensor product CC wavelet family, the center BCC linear box spline wavelet, and the right is the BCC 2,1 wavelet. We removed most of the detail coefficients to amplify the appearance of compression artifacts.
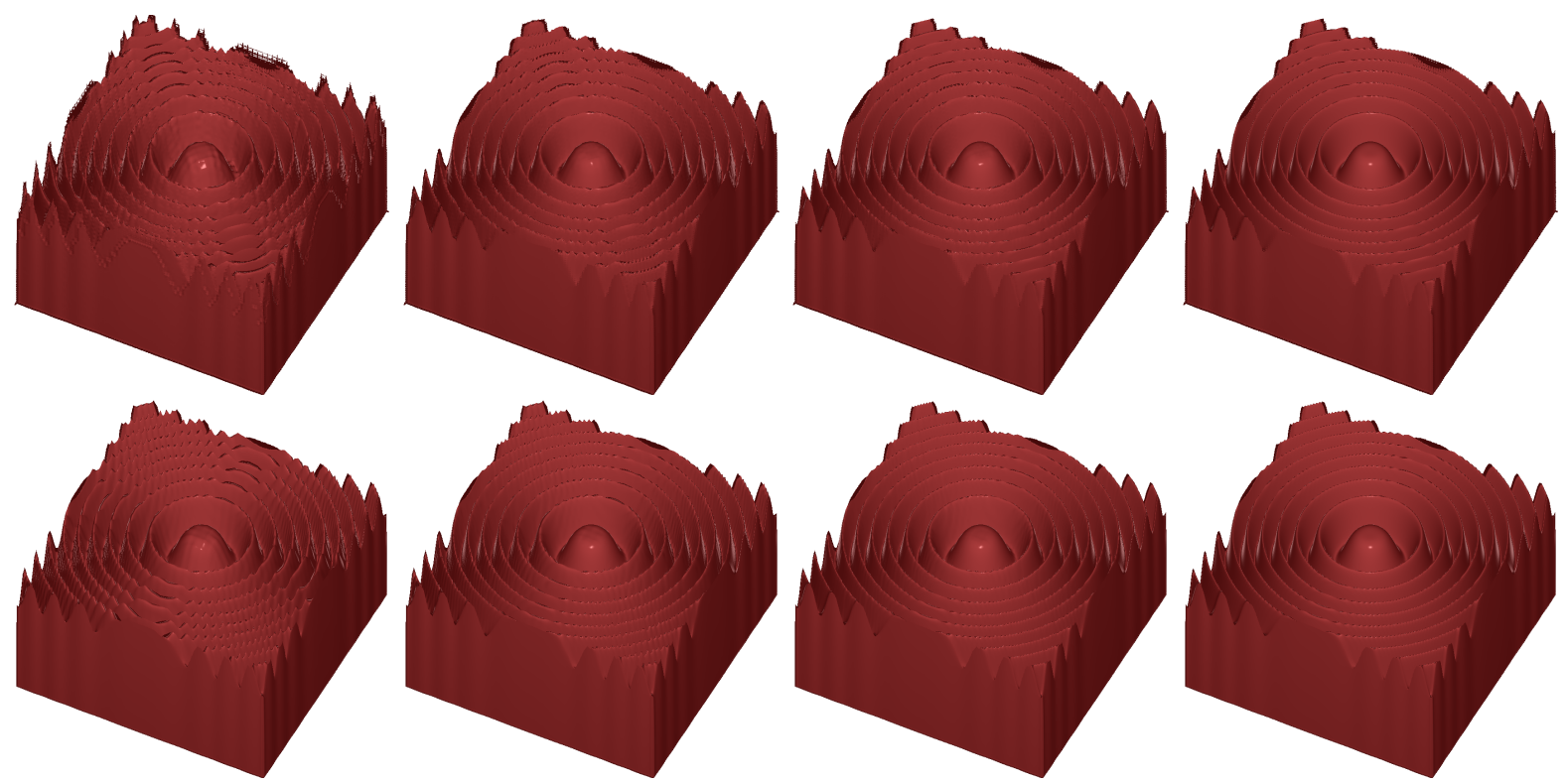

Figure 8: Reconstructions of the synthetic Marschner Lobb function at 5\%, 15\%, 25\% and 100\% of detail coefficients, left to right, respectively. The top row are the reconstructions on the BCC lattice, where the bottom row are reconstructions on the CC lattice. 

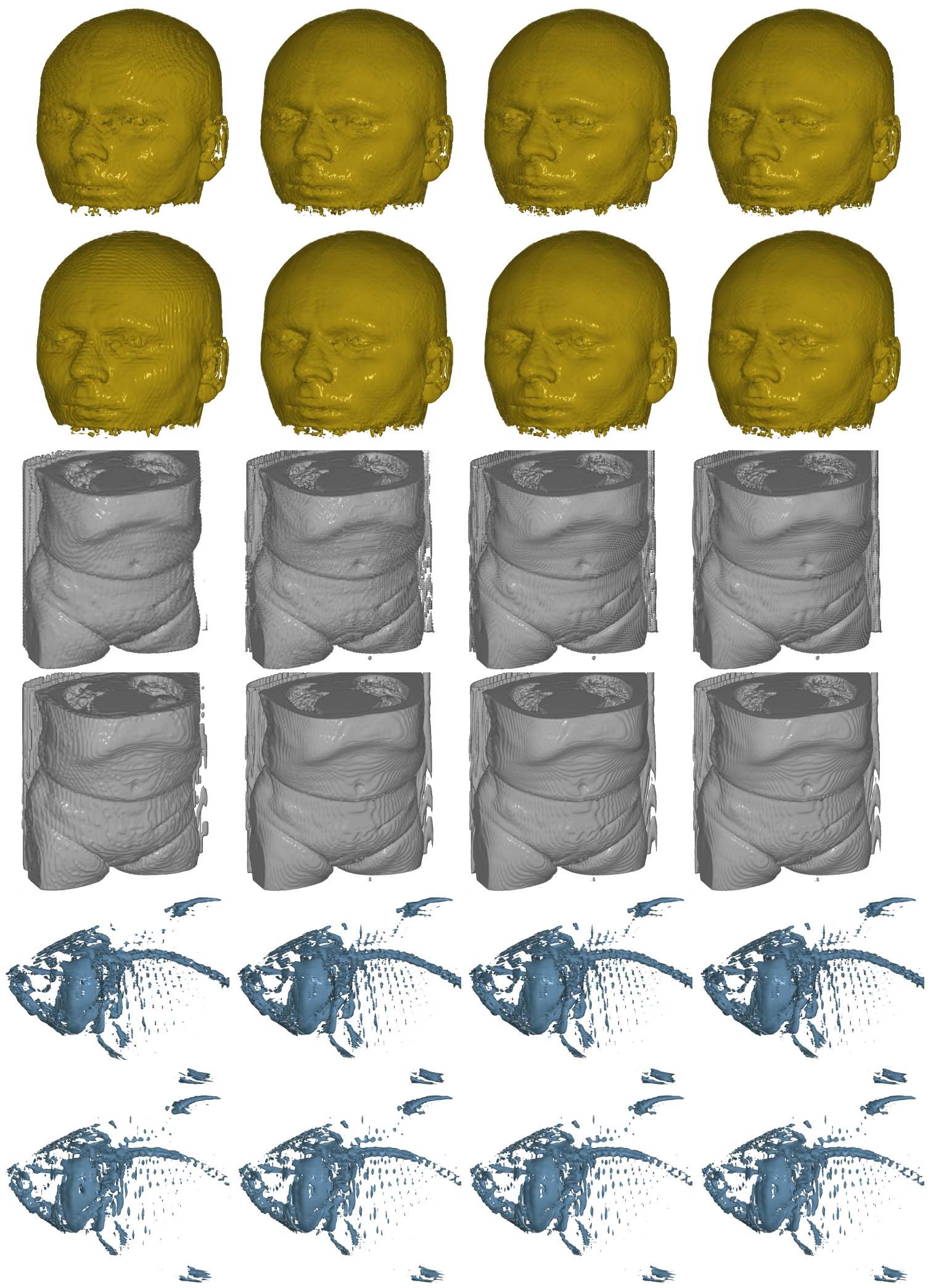

Figure 9: Reconstructions of the test data with 5\%,15\%, 25\% and 100\% of the detail coefficients, left to right, respectively. Within each pair of rows, the top row are the reconstructions on the BCC lattice, whereas the bottom are the reconstructions on the CC lattice. 


\section{J. J. Horacsek \& U. R. Alim / BCC Wavelets}

\section{References}

[AEM09] Alim U. R., Entezari A., Möller T.: The lattice-Boltzmann method on optimal sampling lattices. IEEE Transactions on Visualization and Computer Graphics 15, 4 (07 2009), 630-641. 2, 6

[AM09] Alim U. R., MÖLleR T.: A fast Fourier transform with rectangular output on the BCC and FCC lattices. In Eighth International Conference on Sampling Theory and Applications (SampTA'09) (Marseille, France, 05 2009). 2

[AMC10] Alim U. R., MÖLler T., CONDAT L.: Gradient estimation revitalized. IEEE Transactions on Visualization and Computer Graphics (Proceedings Visualization / Information Visualization 2010) 16, 6 (11 2010), 1494-1503. 2

[AO15] Alim U. R., OliveIRA T. V. D.: A quality-preserving Cartesian to body-centered cubic downsampling transform. In Eurographics Conference on Visualization - Short Papers (2015), The Eurographics Association. 2

[BTU01] Blu T., ThÉVEnAZ P., UnSER M.: MOMS: Maximal-order interpolation of minimal support. IEEE Transactions on Image Processing 10, 7 (2001), 1069-1080. 6

[CDF92] Cohen A., Daubechies I., Feauveau J.-C.: Biorthogonal bases of compactly supported wavelets. Communications on pure and applied mathematics 45, 5 (1992), 485-560. 7

[CS93] COHEN A., SCHLEnKer J. M.: Compactly supported bidimensional wavelet bases with hexagonal symmetry. Constructive Approximation 9, 2 (1993), 209-236. 1, 2, 3, 5, 6

[Csé13] CsÉBFALVI B.: Cosine-weighted B-spline interpolation: A fast and high-quality reconstruction scheme for the body-centered cubic lattice. IEEE transactions on visualization and computer graphics 19, 9 (2013), 1455-1466. 2

[CSW92] Chui C. K., STÖCKLER J., WARD J. D.: Compactly supported box-spline wavelets. Approximation Theory and its Applications 8, 3 (1992), 77-100. 1

[DBHR13] De Boor C., Höllig K., Riemenschneider S.: Box splines, vol. 98. Springer Science \& Business Media, 2013. 3, 4

[DC10] Domonkos B., CsÃL'BFALVI B.: DC-splines: Revisiting the trilinear interpolation on the body-centered cubic lattice. In Vision, Modeling, and Visualization (2010), The Eurographics Association. 2

[EDM04] ENTEZARI A., DYER R., MöLler T.: Linear and cubic box splines for the body centered cubic lattice. In IEEE Visualization 2004 (10 2004), pp. 11-18. 2, 4

[EMBM06] Entezari A., Meng T., Bergner S., Möller T.: A granular three dimensional multiresolution transform. In Proceedings of the Eighth Joint Eurographics / IEEE VGTC Conference on Visualization (2006), Eurographics Association, pp. 267-274. 2

[EMV04] Entezari A., Möller T., VAisey J.: Subsampling matrices for wavelet decompositions on body centered cubic lattices. IEEE Signal Processing Letters 11, 9 (2004), 733-735. 2

[EVDVM08] Entezari A., Van De Ville D., Möeller T.: Practical box splines for reconstruction on the body centered cubic lattice. IEEE Transactions on Visualization and Computer Graphics 14, 2 (2008), 313-328. 2

[FAVM09] Finkbeiner B., Alim U. R., Ville D. V. D., Möller T.: High-quality volumetric reconstruction on optimal lattices for computed tomography. Computer Graphics Forum (Proceedings of Eurographics/IEEE-VGTC Symposium on Visualization 2009) 28, 3 (06 2009), 1023-1030. 2
[HAM11] Hossain Z., Alim U. R., Möller T.: Toward high quality gradient estimation on regular lattices. IEEE Transactions on Visualization and Computer Graphics 17, 4 (04 2011), 426-439. 2

[HK15] HyUnJUN KIM M. K.: Surface Reconstruction from Oriented Point Cloud Using a Box-Spline on the BCC Lattice. Journal of the Korea Computer Graphics Society 21 (2015), 1-10. 2

[HL03] HE W., LAI M.-J.: Construction of trivariate compactly supported biorthogonal box spline wavelets. Journal of Approximation Theory 120, 1 (2003), 1 - 19. 3, 5, 6

[Hor17] HORACSEK J. J.: BCC wavelets. https://github.com/ jjh13/bcc-wavelet, 2017. 8

[Jia99] JIA R.-Q.: Characterization of smoothness of multivariate refinable functions in Sobolev spaces. Transactions of the American Mathematical Society 351, 10 (1999), 4089-4112. 3

[JJL02] JIA R.-Q., JIANG Q., LEE S.: Convergence of cascade algorithms in Sobolev spaces and integrals of wavelets. Numerische Mathematik 91, 3 (2002), 453-473. 3, 6

[JRS99] Ji H., RIEMENSCHNEIDER S. D., SHEN Z.: Multivariate compactly supported fundamental refinable functions, duals, and biorthogonal wavelets. Studies in Applied Mathematics 102, 2 (1999), 173-204. 6

[Kim13] KIM M.: Quartic box-spline reconstruction on the BCC lattice. IEEE transactions on visualization and computer graphics 19, 2 (2013), 319-330. 2

[KV92] KOVAC̆EVIĆ J., VETTERLI M.: Nonseparable multidimensional perfect reconstruction filter banks and wavelet bases for $\mathbb{R}^{n}$. IEEE Transactions on Information Theory 38, 2 (1992), 533-555. 2, 4

[ML94] MARSCHNER S. R., LOBB R. J.: An evaluation of reconstruction filters for volume rendering. In Proceedings of the conference on Visualization'94 (1994), IEEE Computer Society Press, pp. 100-107. 6

[MMZ11a] Matei B., MeIGnen S., ZaKharova A.: Smoothness characterization and stability of nonlinear and non-separable multiscale representations. Journal of Approximation Theory 163, 11 (2011), 1707-1728. 3

[MMZ11b] Matei B., Meignen S., Zakharova A.: Smoothness of nonlinear and non-separable subdivision schemes. Asymptotic Analysis 74, 3-4 (2011), 229-247. 3

[MPS08] Manson J., Petrova G., Schaefer S.: Streaming surface reconstruction using wavelets. In Computer Graphics Forum (2008), vol. 27, Wiley Online Library, pp. 1411-1420. 8

[PK16] PetKov K., Kaufman A. E.: Frameless volume visualization. IEEE Transactions on Visualization and Computer Graphics 22, 2 (02 2016), 10761087. 2

[Ron88] Ron A.: Exponential box splines. Constructive Approximation 4, 1 (1988), 357-378. 2

[The16] The Sage DeVElopers: SageMath, the Sage Mathematics Software System (Version 7.2), 2016. http://www.sagemath.org. 6

[VCRG14] VAD V., CsÉbFalvi B., RAuteK P., Gröller E.: Towards an unbiased comparison of $\mathrm{CC}, \mathrm{BCC}$, and FCC lattices in terms of prealiasing. vol. 33, pp. 81-90. 7

[YE12] YE W., ENTEZARI A.: Design of bivariate sinc wavelets. In Image Processing (ICIP), 2012 19th IEEE International Conference on (2012), IEEE pp. 2477-2480. 3 